\title{
Généralisation d'une famille de Shanks
}

\author{
par \\ BrigitTe AdAm (Metz)
}

1. Introduction. Un problème classique de la théorie des nombres est la recherche de familles paramétrées infinies de corps de nombres algébriques dont un système fondamental d'unités s'écrit simplement en fonction des paramètres.

En degré 2, Shanks [10] a donné des familles paramétrées de corps quadratiques réels $\mathbb{Q}(\alpha)$ pour lesquelles l'unité fondamentale $\varepsilon$ est particulièrement simple, c'est-à-dire de la forme $\varepsilon=\alpha \beta^{n}$, alors que la longueur du développement en fraction continue de $\alpha$ est linéaire en $n$. Ces familles ont été généralisées par plusieurs auteurs, dont Halter-Koch [6], Mollin et Williams [8], [12], qui introduisent de nouveaux paramètres.

En degré 3, C. Levesque et G. Rhin [7] ont étudié le développement par l'AJP de deux familles infinies de corps de nombres algébriques cubiques non totalement réels dépendant chacune de deux paramètres. L'une de ces familles a également été étudiée par A. Farhane [5], [4] qui, le premier, a montré la fondamentalité de l'unité conjecturée par C. Levesque et G. Rhin lorsque l'un des paramètres est suffisamment grand. Dans [1] nous avons étudié leur développement par l'algorithme de Voronoï et obtenu l'unité fondamentale de ces corps. Nous pouvons considérer ces familles comme des analogues de celle de Shanks en degré 3 .

Dans cet article, nous étudions le corps $K=\mathbb{Q}(\alpha)$ où $\alpha$ est la seule racine réelle du polynôme

$$
f(X)=X^{3}-r c^{m} X^{2}-\left(c^{t}-1\right) X-r c^{m}
$$

avec $c \geq 2, m \geq 1,1 \leq t \leq m$ et $r$ divise $c^{\operatorname{pgcd}(m, t)}-1$.

Nous généralisons ainsi les familles précédentes, qui correspondent à $r=$ $t=1$, et obtenons des résultats semblables, à savoir :

- le développement par l'AJP et celui par l'algorithme de Voronoï sont identiques;

1991 Mathematics Subject Classification: 11Y65, 11A55, 11R27, 11R29. 
- la longueur de ces développements tend vers l'infini;

- l'unité fondamentale de l'ordre $\mathbb{Z}[\alpha]$ s'écrit simplement en fonction des paramètres et est du même type que précédemment.

\section{Rappels sur l'AJP et sur l'algorithme de Voronoï}

\subsection{Algorithme de Jacobi-Perron}

DÉfinition 2.1. Soit $\alpha=\left(\alpha_{1}, \ldots, \alpha_{n}\right)$ un vecteur de $\mathbb{R}^{n}(n \geq 1)$. Le développement par l'algorithme de Jacobi-Perron [9] (noté AJP) de $\alpha$ est la donnée :

- d'une suite de vecteurs $\left(a^{(\nu)}\right)_{\nu \geq 0}$ de $\mathbb{Z}^{n}$ appelés quotients incomplets, notés $a^{(\nu)}=\left(a_{1}^{(\nu)}, \ldots, a_{n}^{(\nu)}\right)$;

- d'une suite de vecteurs $\left(\alpha^{(\nu)}\right)_{\nu \geq 0}$ de $\mathbb{R}^{n}$ appelés quotients complets, notés $\alpha^{(\nu)}=\left(\alpha_{1}^{(\nu)}, \ldots, \alpha_{n}^{(\nu)}\right)$

définis par :

- $\alpha^{(0)}=\alpha$

- $\operatorname{pour} \nu \geq 0, a_{i}^{(\nu)}=\left[\alpha_{i}^{(\nu)}\right]$ pour $1 \leq i \leq n$;

- $\operatorname{si} \alpha_{1}^{(\nu)} \neq a_{1}^{(\nu)}$,

$$
\alpha_{n}^{(\nu+1)}=\frac{1}{\alpha_{1}^{(\nu)}-a_{1}^{(\nu)}} ; \quad \alpha_{i}^{(\nu+1)}=\frac{\alpha_{i+1}^{(\nu)}-a_{i+1}^{(\nu)}}{\alpha_{1}^{(\nu)}-a_{1}^{(\nu)}} \quad \text { pour } 1 \leq i<n,
$$

où $[x]$ désigne la partie entière du réel $x$.

DÉFINITION 2.2. Le développement par l'AJP est dit périodique s'il existe deux nombres entiers $k$ positif et $l$ strictement positif tels que $a_{i}^{(k+\nu)}=$ $a_{i}^{(k+\nu+l)}$ pour $\nu \geq 0$ et $1 \leq i \leq n$.

Si $k$ et $l$ sont les plus petits entiers vérifiant cette égalité, alors $k$ est la longueur de la prépériode et $l$ la longueur de la période du développement. Le développement est dit purement périodique si $k=0$.

Remarque. Lorsque le développement par l'AJP de $\alpha=\left(\alpha_{1}, \ldots, \alpha_{n}\right)$ est purement périodique de longueur $l$, il fournit une unité du corps $K=$ $\mathbb{Q}\left[\alpha_{1}, \ldots, \alpha_{n}\right]$, à savoir $[2]$

$$
\varepsilon=\prod_{\nu=0}^{l-1} \alpha_{n}^{(\nu)}
$$

2.2. Algorithme de Voronö̈

2.2.1. Notations et définitions. Soit $K \subseteq \mathbb{R}$ un corps de nombres cubique à conjugués complexes. Soit $L$ un $\mathbb{Z}$-module libre de rang 3 de $K$ de base $\left\{1, \alpha_{1}, \alpha_{2}\right\}$. On dira que $L$ est un réseau de $K$ et on notera $L=\left\langle 1, \alpha_{1}, \alpha_{2}\right\rangle$. A tout point non nul $P=(u, v, w)$ (respectivement $Q$ ) de $\mathbb{Z}^{3}$ on associe 
l'élément $\psi=\psi(P)=u+v \alpha_{1}+w \alpha_{2}$ (respectivement $\left.\phi=\phi(Q)\right)$ de $L$ et on définit

$$
F(P)=\frac{N(\psi)}{\psi}=\psi^{\prime} \psi^{\prime \prime}
$$

où $N$ désigne la norme de $K$ sur $\mathbb{Q}$ et $\psi^{\prime}$ et $\psi^{\prime \prime}$ les conjugués de $\psi$.

DÉfinition 2.3. On dit que $\psi=\psi(P)$ est un point extrémal de $L$ si et seulement si pour tout $\phi=\phi(Q)$ de $L$ tel que $0<\phi<\psi$ on a $F(Q)>F(P)$.

DÉfinition 2.4. Soit $k$ un entier positif. On dit que $\psi_{k+1}$ est le point extrémal adjacent (à droite) à $\psi_{k}$ dans $L$ si et seulement si

$$
\psi_{k+1}=\min \left\{\psi \text { tel que } \psi>\psi_{k} \text { et } F(P)<F\left(P_{k}\right)\right\} .
$$

On définit ainsi une suite croissante des points extrémaux de $L$ par

$$
\psi_{0}=1, \quad \psi_{k+1} \text { est le point extrémal adjacent à } \psi_{k} \text { si } k \geq 0 \text {. }
$$

On considère un ordre $\mathcal{O}$ de $K$ et $L=\mathcal{O}$. Par Voronoï [3], on sait que la suite précédente est purement périodique de la forme

$$
\overbrace{1=\psi_{0}, \ldots, \psi_{l-1}}, \overbrace{\psi_{l}=\varepsilon, \varepsilon \psi_{1}, \ldots, \varepsilon \psi_{l-1}} \overbrace{\varepsilon^{2}, \ldots, \varepsilon^{2} \psi_{l-1}}, \ldots
$$

où $l$ est la longueur de la période et $\varepsilon$ l'unité fondamentale supérieure à 1 de $\mathcal{O}$.

2.2.2. Méthode de construction de points extrémaux. Pour construire une telle suite il suffit de savoir construire le point extrémal adjacent à 1 dans un réseau $L=\left\langle 1, \alpha_{1}, \alpha_{2}\right\rangle$.

En effet, soit $\psi_{0}=1$ et $\psi_{1}$ le point extrémal adjacent à 1 dans $L_{0}=\mathcal{O}=$ $\left\langle 1, \alpha_{1}, \alpha_{2}\right\rangle$.

(a) On choisit un point auxiliaire $\phi_{1}$ tel que $\left\{\psi_{1}, \phi_{1}, \psi_{0}\right\}$ soit une base de $L_{0}$.

(b) Dire que $\psi_{2}$ est le point extrémal adjacent à $\psi_{1}$ dans $\mathcal{L}_{1}=\left\langle\psi_{1}, \phi_{1}, \psi_{0}\right\rangle$ équivaut à dire que $\psi_{2} / \psi_{1}$ est le point extrémal adjacent à 1 dans $L_{1}=\left\langle 1, \phi_{1} / \psi_{1}, \psi_{0} / \psi_{1}\right\rangle$.

On itère ce processus.

Ainsi on cherche un élément $\psi=u+v \alpha_{1}+w \alpha_{2}$ de $L=\left\langle 1, \alpha_{1}, \alpha_{2}\right\rangle$ tel que

$$
\psi>1, \quad F(P)<1, \quad \psi \text { minimum. }
$$

En notant $F(u, v, w)=\psi^{\prime} \psi^{\prime \prime}, F$ définit une forme quadratique à trois variables $u, v, w$ à coefficients réels, positive de rang 2 . On va rappeler ici une proposition [1] qui, utilisant un vecteur isotrope de cette forme quadratique, nous permet de restreindre à 5 au maximum le nombre de choix pour un point extrémal adjacent à 1 . 
On supposera dans la suite que $\left(\gamma_{2}, 1, \gamma_{1}\right)$ est un vecteur isotrope de $F$ et on pose

$$
\begin{aligned}
& \phi_{1}=\left[\gamma_{2}\right]+\alpha_{1}, \quad Q_{1}=\left(\left[\gamma_{2}\right], 1,0\right), \\
& \phi_{2}=\left[\gamma_{2}\right]+\alpha_{1}+\alpha_{2}, \quad Q_{2}=\left(\left[\gamma_{2}\right], 1,1\right), \\
& \phi_{3}=\left[\gamma_{2}\right]+\alpha_{1}-\alpha_{2}, \quad Q_{3}=\left(\left[\gamma_{2}\right], 1,-1\right), \\
& \phi_{4}=\left[\gamma_{2}\right]-1+\alpha_{1}, \quad Q_{4}=\left(\left[\gamma_{2}\right]-1,1,0\right), \\
& \phi_{5}=\left[\gamma_{2}\right]-1+\alpha_{1}+\alpha_{2}, \quad Q_{5}=\left(\left[\gamma_{2}\right]-1,1,1\right) \text {, } \\
& \phi_{6}=\left[\gamma_{2}\right]+1+2 \alpha_{1}-\alpha_{2}, \quad Q_{6}=\left(\left[\gamma_{2}\right]+1,2,-1\right) \text {, } \\
& \phi_{7}=\left[\gamma_{2}\right]+2 \alpha_{1}, \quad Q_{7}=\left(\left[\gamma_{2}\right], 2,0\right), \\
& \phi_{8}=\left[\gamma_{2}\right]+1+\alpha_{1}-\alpha_{2}, \quad Q_{8}=\left(\left[\gamma_{2}\right]+1,1,-1\right) \text {. }
\end{aligned}
$$

LEMme 2.5. Soit $F$ une forme quadratique à trois variables $u, v, w, \grave{a}$ coefficients réels, positive de rang 2 telle que

$$
F(1,0,0)=1 \text { et } F(0,0,1)>1 .
$$

Si $F$ admet un vecteur isotrope $\left(\gamma_{2}, 1, \gamma_{1}\right)$ alors $F$ s'écrit

$$
F(u, v, w)=a\left(w-\gamma_{1} v\right)^{2}+2 b\left(w-\gamma_{1} v\right)\left(u-\gamma_{2} v\right)+\left(u-\gamma_{2} v\right)^{2}
$$

$e t$

(3) $\quad F(u, v, w)$

$$
=\frac{a}{2}\left[w-\left(\gamma_{1}+2 \frac{b}{a} \gamma_{2}\right) v+2 \frac{b}{a} u\right]^{2}+\frac{a}{2}\left(w-\gamma_{1} v\right)^{2}+\left(1-2 \frac{b^{2}}{a}\right)\left(u-\gamma_{2} v\right)^{2}
$$

avec $a>1$ et $b^{2}<a$.

Proposition 2.6 [1]. Soient $0<\gamma_{1}<1, \gamma_{2}>1,0<\alpha_{1}<1,0<\alpha_{2}<1$ et $4 b^{2}<a$.

1. Si $F\left(Q_{1}\right)<1$,

(a) si $b<0$ alors le point extrémal adjacent à 1 est $\phi_{1}, \phi_{3}$ ou $\phi_{4}$;

(b) si $b \geq 0$ alors le point extrémal adjacent à 1 est $\phi_{1}$ ou $\phi_{5}$.

2. Si $F\left(Q_{1}\right)>1$ et $F\left(Q_{2}\right)<1$,

(a) si $b<0$ alors le point extrémal adjacent à 1 est:

(i) $\phi_{2}, \phi_{3}$ ou $\phi_{4}$ si $\alpha_{2}<\alpha_{1}$;

(ii) $\phi_{2}, \phi_{3}, \phi_{4}$ ou $\phi_{7}$ si $2 \alpha_{2}-1<\alpha_{1}<\alpha_{2}$;

(iii) $\phi_{2}, \phi_{3}, \phi_{4}, \phi_{6}$ ou $\phi_{7}$ si $\alpha_{1}<2 \alpha_{2}-1$;

(b) si $b \geq 0$ alors le point extrémal adjacent à 1 est:

(i) $\phi_{2}$ ou $\phi_{5}$ si $2 \alpha_{2}-1<0$;

(ii) $\phi_{2}, \phi_{5}$ ou $\phi_{8}$ si $2 \alpha_{2}-1>0$. 
Cette proposition va nous permettre de donner explicitement la suite croissante des points extrémaux du développement par l'algorithme de Voronoï pour les familles étudiées par la suite.

3. Etude d'une première famille. Soient $c \geq 2, m \geq 1$ et $1 \leq t \leq m$ des entiers. On considère le polynôme

$$
f(X)=X^{3}-c^{m} X^{2}-\left(c^{t}-1\right) X-c^{m} .
$$

On montre facilement que $f(X)$ est irréductible et admet une racine réelle unique notée $\alpha$ telle que $c^{m}<\alpha<c^{m}+1$.

On note $K=\mathbb{Q}(\alpha), \mathcal{O}=\mathbb{Z}[\alpha], \alpha_{2}=\alpha, \alpha_{1}=\alpha\left(\alpha-c^{m}\right)$.

3.1. Développement par l'algorithme de Jacobi-Perron. On a le théorème suivant :

ThÉORÈme 3.1. Le développement par l'AJP de $\left(\alpha_{1}, \alpha_{2}\right)$ est purement périodique de longueur $l=(3 m+t) / d$ où $d=\operatorname{pgcd}(m, t)$ et

$$
\varepsilon=\alpha^{t / d}\left(\frac{\alpha}{\alpha-c^{m}}\right)^{m / d}
$$

est une unité de $\mathbb{Z}[\alpha]$.

3.1.1. Lemmes utiles à la démonstration du théorème

LeMme 3.2. Si au rang $k, \alpha_{2}^{(k)}=\alpha / c^{l}$ et $\alpha_{1}^{(k)}=\alpha\left(\alpha-c^{m}\right) / c^{l}$ avec l un entier tel que $0<l<t$ alors

$$
\alpha_{2}^{(k+4)}=\frac{\alpha}{c^{l+m-t}} \quad \text { et } \quad \alpha_{1}^{(k+4)}=\frac{\alpha\left(\alpha-c^{m}\right)}{c^{l+m-t}}
$$

et on a les résultats suivants :

$$
\begin{aligned}
& \left\{\begin{array}{l}
a_{2}^{(k)}=c^{m-l}, \\
a_{1}^{(k)}=c^{t-l}-1,
\end{array}\right. \\
& \begin{cases}\alpha_{2}^{(k+1)}=\frac{\alpha c^{l}}{\alpha\left(c^{l}-1\right)+c^{m}}, & a_{2}^{(k+1)}=1, \\
\alpha_{1}^{(k+1)}=\frac{\alpha\left(\alpha-c^{m}\right)}{\alpha\left(c^{l}-1\right)+c^{m}}, & a_{1}^{(k+1)}=0,\end{cases} \\
& \begin{cases}\alpha_{2}^{(k+2)}=\frac{\alpha\left(c^{l}-1\right)+c^{m}}{\alpha\left(\alpha-c^{m}\right)}, & a_{2}^{(k+2)}=c^{l+m-t}, \\
\alpha_{1}^{(k+2)}=\frac{1}{\alpha}, & a_{1}^{(k+2)}=0,\end{cases} \\
& \begin{cases}\alpha_{2}^{(k+3)}=\alpha, & a_{2}^{(k+3)}=c^{m}, \\
\alpha_{1}^{(k+3)}=\frac{\alpha\left(c^{l}-1\right)+c^{l+m-t}}{\alpha}, & a_{1}^{(k+3)}=c^{l}-1 .\end{cases}
\end{aligned}
$$


Preuve. Les inégalités $c^{m}<\alpha<c^{m}+1$ et $c^{t}-1<\alpha\left(\alpha-c^{m}\right)<c^{t}$ impliquent que $a_{2}^{(k)}=c^{m-l}$ et $a_{1}^{(k)}=c^{t-l}-1$. On en déduit facilement $\alpha_{2}^{(k+1)}$ et $\alpha_{1}^{(k+1)}$.

On a

$$
\alpha_{2}^{(k+1)}-1=\frac{\alpha-c^{m}}{\alpha\left(c^{l}-1\right)+c^{m}}>0 \quad \text { et } \quad 2-\alpha_{2}^{(k+1)}=\frac{\alpha c^{l}-2\left(\alpha-c^{m}\right)}{\alpha\left(c^{l}-1\right)+c^{m}}>0,
$$

donc $a_{2}^{(k+1)}=1$. On a $\alpha\left(c^{l}-1\right)+c^{m}>c^{t}$ et $\alpha\left(\alpha-c^{m}\right)<c^{t}$, donc $a_{1}^{(k+1)}=0$. On en déduit $\alpha_{2}^{(k+2)}$ et $\alpha_{1}^{(k+2)}$.

Montrons que $a_{2}^{(k+2)}=c^{l+m-t}$ : on a

$$
\begin{aligned}
\alpha_{2}^{(k+2)}-c^{l+m-t} & =\frac{1}{\alpha\left(\alpha-c^{m}\right)}\left(\alpha\left(c^{l}-1\right)+c^{m}-c^{l+m-t}\left(c^{t}-1+\frac{c^{m}}{\alpha}\right)\right) \\
& =\frac{1}{\alpha\left(\alpha-c^{m}\right)}\left(\left(\alpha-c^{m}\right)\left(c^{l}-1\right)+c^{l+m-t} \frac{\alpha-c^{m}}{\alpha}\right)>0,
\end{aligned}
$$

et

$$
\begin{aligned}
c^{l+m-t}+1-\alpha_{2}^{(k+2)}= & \frac{1}{\alpha\left(\alpha-c^{m}\right)}\left(c^{l+m-t}\left(c^{t}-1+\frac{c^{m}}{\alpha}\right)\right. \\
& \left.+\alpha\left(\alpha-c^{m}\right)-\alpha\left(c^{l}-1\right)-c^{m}\right) \\
= & \frac{1}{\alpha}\left(\alpha-c^{l}+\frac{\alpha-c^{l+m-t}}{\alpha}\right)>0,
\end{aligned}
$$

d'où le résultat.

$a_{1}^{(k+2)}=0$ de façon évidente et on en déduit $\alpha_{2}^{(k+3)}$ et $\alpha_{1}^{(k+3)}$.

On a

$$
c^{l}-1=\frac{\alpha\left(c^{l}-1\right)}{\alpha}<\alpha_{1}^{(k+3)}<\frac{\alpha c^{l}}{\alpha}=c^{l},
$$

donc $a_{1}^{(k+2)}=c^{l}-1$. On a $a_{2}^{(k+3)}=c^{m}$ de façon évidente et on en déduit $\alpha_{2}^{(k+4)}$ et $\alpha_{1}^{(k+4)}$; ce qui termine la démonstration du lemme 3.2.

LEMME 3.3. Si au rang $k, \alpha_{2}^{(k)}=\alpha / c^{l}$ et $\alpha_{1}^{(k)}=\alpha\left(\alpha-c^{m}\right) / c^{l}$ avec $l$ un entier tel que $t \leq l \leq m$ alors $\alpha_{2}^{(k+3)}=\alpha / c^{l-t}$ et $\alpha_{1}^{(k+3)}=\alpha\left(\alpha-c^{m}\right) / c^{l-t}$ et on a les résultats suivants :

$$
\begin{aligned}
& \left\{\begin{array}{l}
a_{2}^{(k)}=c^{m-l}, \\
a_{1}^{(k)}=0,
\end{array}\right. \\
& \begin{cases}\alpha_{2}^{(k+1)}=\frac{c^{l}}{\alpha\left(\alpha-c^{m}\right)}, & a_{2}^{(k+1)}=c^{l-t}, \\
\alpha_{1}^{(k+1)}=\frac{1}{\alpha}, & a_{1}^{(k+1)}=0,\end{cases}
\end{aligned}
$$




$$
\begin{cases}\alpha_{2}^{(k+2)}=\alpha, & a_{2}^{(k+2)}=c^{m}, \\ \alpha_{1}^{(k+2)}=\frac{c^{l-t}}{\alpha}, & a_{1}^{(k+2)}=0 .\end{cases}
$$

Preuve. La démonstration est semblable à celle du lemme précédent. On montre uniquement que $a_{2}^{(k+1)}=c^{l-t}$ : on a

$$
\begin{aligned}
\alpha_{2}^{(k+1)}-c^{l-t} & =\frac{1}{\alpha\left(\alpha-c^{m}\right)}\left(c^{l}-c^{l-t}\left(c^{t}-1+\frac{c^{m}}{\alpha}\right)\right) \\
& =\frac{c^{l-t}}{\alpha\left(\alpha-c^{m}\right)}\left(\frac{\alpha-c^{m}}{\alpha}\right)=\frac{c^{l-t}}{\alpha^{2}}>0,
\end{aligned}
$$

et

$$
\begin{aligned}
c^{l-t}+1-\alpha_{2}^{(k+1)} & =\frac{1}{\alpha\left(\alpha-c^{m}\right)}\left(c^{l-t}\left(c^{t}-1+\frac{c^{m}}{\alpha}\right)+\alpha\left(\alpha-c^{m}\right)-c^{l}\right) \\
& =\frac{1}{\alpha}\left(\left(\alpha-c^{m}\right)\left(\alpha-\frac{c^{l-t}}{\alpha}\right)\right)>0,
\end{aligned}
$$

d'où le résultat.

3.1.2. Calcul explicite du développement par l'AJP. L'algorithme commence avec :

$$
\begin{aligned}
& \begin{cases}\alpha_{2}^{(0)}=\alpha, & a_{2}^{(0)}=c^{m}, \\
\alpha_{1}^{(0)}=\alpha\left(\alpha-c^{m}\right), & a_{1}^{(0)}=c^{t}-1,\end{cases} \\
& \begin{cases}\alpha_{2}^{(1)}=\frac{\alpha}{c^{m}}, & a_{2}^{(1)}=1, \\
\alpha_{1}^{(1)}=\frac{\alpha\left(\alpha-c^{m}\right)}{c^{m}}, & a_{1}^{(1)}=0 .\end{cases}
\end{aligned}
$$

On note $q$ la partie entière de $m / t$. En appliquant $q$ fois le lemme 3.3 , on obtient

$$
\left\{\begin{array}{l}
\alpha_{2}^{(3 q+1)}=\frac{\alpha}{c^{m-q t}}, \\
\alpha_{1}^{(3 q+1)}=\frac{\alpha\left(\alpha-c^{m}\right)}{c^{m-q t}} .
\end{array}\right.
$$

Soit $n_{0}$ le plus grand entier tel que $\left(n_{0}+1\right) m-\left(n_{0}+q\right) t<t$. En appliquant $n_{0}+1$ fois le lemme 3.2 , on obtient

$$
\left\{\begin{array}{l}
\alpha_{2}^{\left(3 q+1+4\left(n_{0}+1\right)\right)}=\frac{\alpha}{c^{\left(n_{0}+2\right) m-\left(q+n_{0}+1\right) t}}, \\
\alpha_{1}^{\left(3 q+1+4\left(n_{0}+1\right)\right)}=\frac{\alpha\left(\alpha-c^{m}\right)}{c^{\left(n_{0}+2\right) m-\left(q+n_{0}+1\right) t}} .
\end{array}\right.
$$


On applique le lemme 3.3 et on obtient

$$
\left\{\begin{array}{l}
\alpha_{2}^{\left(3 q+1+4\left(n_{0}+1\right)+3\right)}=\frac{\alpha}{c^{\left(n_{0}+2\right) m-\left(q+n_{0}+2\right) t}}, \\
\alpha_{1}^{\left(3 q+1+4\left(n_{0}+1\right)+3\right)}=\frac{\alpha\left(\alpha-c^{m}\right)}{c^{\left(n_{0}+2\right) m-\left(q+n_{0}+2\right) t}} .
\end{array}\right.
$$

Si $\left(n_{0}+2\right) m-\left(q+n_{0}+2\right) t=0$ alors

$$
\left\{\begin{array}{l}
\alpha_{2}^{\left(3 q+1+4\left(n_{0}+1\right)+3\right)}=\alpha_{2}^{(0)}, \\
\alpha_{1}^{\left(3 q+1+4\left(n_{0}+1\right)+3\right)}=\alpha_{1}^{(0)},
\end{array}\right.
$$

ce qui nous donne la période du développement.

Sinon, on considère $n_{1}$ le plus grand entier tel que $\left(n_{0}+n_{1}+2\right) m-(q+$ $\left.n_{0}+n_{1}+2\right) t<t$ et on applique $\left(n_{1}+1\right)$ fois le lemme 3.2.

On poursuit alors ce processus. On va, dans le paragraphe qui suit, montrer que ce processus s'arrête.

3.1.3. Calcul de la longueur du développement. Pour tout entier $k$, on note $n_{k}$ le plus grand entier tel que

$$
\left(n_{0}+\ldots+n_{k}+k+1\right) m-\left(q+n_{0}+\ldots+n_{k}+2 k+1\right) t<0 .
$$

On montre qu'il existe un entier $k$ tel que

$$
\left(n_{0}+\ldots+n_{k}+k+2\right) m-\left(q+n_{0}+\ldots+n_{k}+2 k+2\right) t=0
$$

et on note $k_{0}$ le plus petit entier vérifiant cette relation.

La longueur de la période du développement est alors

$$
l=3 q+1+\left(4\left(n_{0}+1\right)+3\right)+\ldots+\left(4\left(n_{k_{0}}+1\right)+3\right) .
$$

Montrons que $k_{0}$ est bien défini et que $l=(3 m+t) / d$.

Pour tout entier $k, n_{k}$ est défini par le système suivant :

$$
\left\{\begin{array}{l}
\left(n_{0}+\ldots+n_{k}+k+1\right) m-\left(q+n_{0}+\ldots+n_{k}+2 k+1\right) t<0, \\
\left(n_{0}+\ldots+n_{k}+k+2\right) m-\left(q+n_{0}+\ldots+n_{k}+2 k+2\right) t \geq 0,
\end{array}\right.
$$

qui nous donne les inégalités

$$
n_{0}+\ldots+n_{k}+k+1<\frac{(q+k) t}{m-t} \leq n_{0}+\ldots+n_{k}+k+2 .
$$

Ainsi $k_{0}$ est le plus petit entier tel que $\left(q+k_{0}\right) t /(m-t)$ soit entier et $k_{0}$ est donc défini par

$$
q+k_{0}=\frac{\operatorname{ppcm}(t, m-t)}{t}=\frac{m-t}{d} .
$$

Ceci montre l'existence de $k_{0}$ et on a la relation $n_{0}+\ldots+n_{k_{0}}+k_{0}+2=t / d$. On en déduit facilement la longueur de la période du développement, à savoir $l=(3 m+t) / d$. 
3.1.4. Calcul de l'unité. Une unité de $\mathbb{Z}[\alpha]$ est donnée par la formule de Hasse-Bernstein [2], à savoir

$$
\begin{aligned}
\varepsilon & =\prod_{r=0}^{l-1} \alpha_{2}^{(r)}=\alpha\left(\frac{\alpha}{\alpha-c^{m}}\right)^{q}\left(\frac{\alpha^{2}}{\alpha-c^{m}}\right)^{t / d-1}\left(\frac{\alpha}{\alpha-c^{m}}\right)^{k_{0}+1} \\
& =\frac{\alpha^{1+q+2 t / d-2+k_{0}+1}}{\left(\alpha-c^{m}\right)^{q+t / d-1+k_{0}+1}}
\end{aligned}
$$

Les égalités

$$
1+q+2 \frac{t}{d}-2+k_{0}+1=\frac{m+t}{d} \quad \text { et } \quad q+\frac{t}{d}-1+k_{0}+1=\frac{m}{d}
$$

impliquent que

$$
\varepsilon=\alpha^{t / d}\left(\frac{\alpha}{\alpha-c^{m}}\right)^{m / d}
$$

3.2. Développement par l'algorithme de Voronoï. Soit $L=\left\langle 1, \alpha_{1}, \alpha_{2}\right\rangle$ un réseau de $K$ et $\psi$ le point extrémal adjacent à 1 dans $L$. En notant $\psi=u+v \alpha_{1}+w \alpha_{2}$ on a les lemmes suivants :

Lemme 3.4. Pour un entier $l, 0 \leq l \leq m$,

- si $L=\left\langle 1, \alpha-c^{m}, c^{l} / \alpha\right\rangle$ alors $(u, v, w)=\left(c^{m}, 1,0\right)$.

Lemme 3.5. Pour un entier $l, 0 \leq l \leq m-t$,

- si $L=\left\langle 1, c^{l} / \alpha^{2}, 1 / \alpha\right\rangle$ alors $(u, v, w)=\left(c^{l}, 1,0\right)$;

- si

$$
L=\left\langle 1, \frac{\alpha-c^{m}}{c^{l+t}}, \frac{\alpha\left(\alpha-c^{m}\right)}{c^{l+t}}\right\rangle
$$

alors $(u, v, w)=\left(c^{m-l-t}, 1,0\right)$.

Lemme 3.6. Pour un entier $l, m-t<l \leq m$,

- $s i$

$$
L=\left\langle 1, \frac{\alpha\left(c^{l+t-m}-1\right)+c^{l}}{\alpha^{2}}, \frac{1}{\alpha}\right\rangle
$$

alors $(u, v, w)=\left(c^{l}, 1,0\right)$;

- $s i$

$$
L=\left\langle 1, \frac{\alpha-c^{m}}{\alpha\left(c^{l+t-m}-1\right)+c^{m}}, \frac{\alpha\left(\alpha-c^{m}\right)}{\alpha\left(c^{l+t-m}-1\right)+c^{m}}\right\rangle
$$

alors $(u, v, w)=(1,1,0)$;

- si

$$
L=\left\langle 1, \frac{\alpha-c^{m}}{c^{l+t-m}}, \frac{\alpha\left(c^{l+t-m}-1\right)+c^{m}}{c^{l+t-m} \alpha}\right\rangle
$$

alors $(u, v, w)=\left(c^{2 m-(l+t)}, 1,0\right)$. 
Ces lemmes se démontrent à l'aide de la proposition 2.6. Ils sont semblables à ceux énoncés dans [1] et les démonstrations en sont identiques. Pour cette raison nous ne donnerons ici, qu'à titre d'exemple, la démonstration du lemme 3.4.

Preuve du lemme 3.4. On vérifie dans ce cas que $F$ est une forme quadratique positive de rang 2 qui s'écrit à l'aide des formules (2) et (3) avec

$$
a=\frac{\alpha}{c^{m-2 l}}, \quad b=-\frac{\alpha\left(\alpha-c^{m}\right)}{2 c^{m-l}}, \quad \omega_{2}=\alpha, \quad \omega_{1}=\frac{c^{m-l}}{\alpha} .
$$

On a $0<\omega_{1}<1, \omega_{2}>1,0<\alpha_{1}<1,0<\alpha_{2}<1$ et $4 b^{2}<a$. En reprenant les notations du paragraphe 2 , on a $\phi_{1}=\alpha$ donc

$$
F\left(Q_{1}\right)=\frac{N(\alpha)}{\alpha}=\frac{c^{m}}{\alpha}<1 \text { et } b<0 .
$$

D'après la proposition 2.6, le point extrémal adjacent à 1 est $\phi_{1}, \phi_{3}$ ou $\phi_{4}$.

Or $Q_{3}=\left(c^{m}, 1,-1\right)$ et l'on a, d'après $(3)$,

$$
F\left(Q_{3}\right)>\frac{\alpha}{2 c^{m-2 l}}\left(1+\frac{c^{m-l}}{\alpha}\right)^{2}>\frac{\alpha}{2 c^{m-2 l}}+c^{l}+\frac{c^{m}}{2 \alpha}>c^{l} \geq 1 .
$$

Enfin $\phi_{4}=\alpha-1$ et

$$
F\left(Q_{4}\right)=\frac{N(\alpha-1)}{\alpha-1}=\frac{2 c^{m}+c^{t}-2}{\alpha-1}>\frac{2 c^{m}+c^{t}-2}{c^{m}}>1 .
$$

Donc $\psi=\phi_{1}$ c'est-à-dire $(u, v, w)=\left(c^{m}, 1,0\right)$.

Ainsi le lemme 3.4 est démontré.

A l'aide de ces lemmes, on détermine la suite des points extrémaux de $\mathcal{O}$ de la façon suivante :

Soit $L_{0}=\left\langle 1, \alpha-c^{m}, c^{m} / \alpha\right\rangle$. Par le lemme 3.4 on a $\psi_{1}=\alpha$. On choisit un point auxiliaire $\phi_{1}$ tel que $\left\{\psi_{1}, \phi_{1}, \psi_{0}\right\}$ soit une base de $L_{0}$, à savoir $\phi_{1}=\alpha\left(\alpha-c^{m}\right)$. Pour déterminer $\psi_{2}$ on cherche le point extrémal adjacent à 1 dans le réseau $L_{1}=\left\langle 1, \phi_{1} / \psi_{1}, \psi_{0} / \psi_{1}\right\rangle=\left\langle 1, \alpha-c^{m}, 1 / \alpha\right\rangle$ et par le même lemme 3.4 on a $\psi_{2} / \psi_{1}=\alpha$, c'est-à-dire $\psi_{2}=\alpha^{2}$.

En poursuivant ce processus, on obtient la suite des points extrémaux de $\mathcal{O}$. Nous donnons dans le tableau 1 , pour $0 \leq s \leq q-1$ (où $q$ désigne la partie entière de $m / t$ ) les premiers résultats obtenus.

On a noté

$$
\phi_{0}=\alpha-c^{m}, \quad \psi_{-1}=\frac{c^{m}}{\alpha}
$$

et les troisième et quatrième colonnes donnent les coordonnées de $\psi_{k+1} / \psi_{k}$ et de $\phi_{k+1} / \psi_{k}$ dans le réseau $L_{k}$. A l'aide des quotients successifs $\psi_{k+1} / \psi_{k}$ on peut facilement déterminer la suite des points extrémaux $\psi_{k}$ de $\mathbb{Z}[\alpha]$. 
Tableau 1

\begin{tabular}{cccc}
\hline$k$ & $L_{k}=\left\langle 1, \phi_{k} / \psi_{k}, \psi_{k-1} / \psi_{k}\right\rangle$ & $\psi_{k+1} / \psi_{k}$ & $\phi_{k+1} / \psi_{k}$ \\
\hline 0 & $\left\langle 1, \alpha-c^{m}, c^{m} / \alpha\right\rangle$ & $\left(c^{m}, 1,0\right)$ & $(c-1,0,1)$ \\
$\vdots$ & $\vdots$ & $\vdots$ & $\vdots$ \\
$3 s+1$ & $\left\langle 1, \alpha-c^{m}, c^{s t} / \alpha\right\rangle$ & $\left(c^{m}, 1,0\right)$ & $(0,0,1)$ \\
$3 s+2$ & $\left\langle 1, c^{s t} / \alpha^{2}, 1 / \alpha\right\rangle$ & $\left(c^{s t}, 1,0\right)$ & $(0,0,1)$ \\
$3 s+3$ & $\left\langle 1,\left(\alpha-c^{m}\right) / c^{(s+1) t}, \alpha\left(\alpha-c^{m}\right) / c^{(s+1) t}\right\rangle$ & $\left(c^{m-(s+1) t}, 1,0\right)$ & $(0,0,1)$ \\
$\vdots$ & $\vdots$ & $\vdots$ & $\vdots$ \\
$3 q+1$ & $\left\langle 1, \alpha-c^{m}, c^{q t} / \alpha\right\rangle$ & $\left(c^{m}, 1,0\right)$ & $\left(c^{(q+1) t-m}-1,0,1\right)$ \\
$3 q+2$ & $\left\langle 1, \frac{\alpha\left(c^{(q+1) t-m}-1\right)+c^{q t}}{\alpha^{2}}, \frac{1}{\alpha}\right\rangle$ & $\left(c^{q t}, 1,0\right)$ & $(0,0,1)$ \\
$3 q+3\left\langle 1, \frac{\alpha-c^{m}}{\alpha\left(c^{(q+1) t-m}-1\right)+c^{m}}, \frac{\alpha\left(\alpha-c^{m}\right)}{\alpha\left(c^{(q+1) t-m}-1\right)+c^{m}}\right.$ & $(1,1,0)$ & $(0,0,1)$ \\
$3 q+4$ & $\left\langle 1, \frac{\alpha-c^{m}}{c^{(q+1) t-m}}, \frac{\alpha\left(c^{(q+1) t-m}-1\right)+c^{m}}{c^{(q+1) t-m}}\right\rangle$ & $\left(c^{2 m-(q+1) t}, 1,0\right)$ & $\left(c^{m-q t}, 0,1\right)$ \\
$\vdots$ & $\vdots$ & $\vdots$ & $\vdots$ \\
\hline
\end{tabular}

On en déduit que

$$
\psi_{(3 m+t) / d}=\alpha^{t / d}\left(\frac{\alpha}{\alpha-c^{m}}\right)^{m / d} .
$$

On a $N\left(\psi_{(3 m+t) / d}\right)=1$ et $N\left(\psi_{i}\right) \neq 1$ si $0<i<(3 m+t) / d$. Donc $\psi_{(3 m+t) / d}$ est l'unité fondamentale supérieure à 1 de $\mathcal{O}$ et la longueur de la période du développement de l'algorithme de Voronoï est $l=(3 m+t) / d$.

On peut remarquer que, de même que pour la famille étudiée dans [1] qui correspond à $t=1$ :

- les points auxiliaires choisis sont donnés par l'AJP;

- les développements par l'AJP et par l'algorithme de Voronoï coïncident : ils ont donc la même longueur et l'unité obtenue par l'AJP est l'unité fondamentale supérieure à 1 de $\mathcal{O}$.

4. Etude d'une deuxième famille. Soit $c \geq 2, m \geq 1$ et $r$ des entiers tel que $r \neq 1$ et $r$ divise $c-1$. On considère le polynôme

$$
f(X)=X^{3}-r c^{m} X^{2}-(c-1) X-r c^{m} .
$$

On montre facilement que $f(X)$ est irréductible et admet une racine réelle unique notée $\alpha$ telle que $r c^{m}<\alpha<r c^{m}+1$. On note $K=\mathbb{Q}(\alpha), \mathcal{O}=\mathbb{Z}[\alpha]$, $\alpha_{2}=\alpha, \alpha_{1}=\alpha\left(\alpha-r c^{m}\right)$.

4.1. Développement par l'algorithme de Jacobi-Perron. On a le théorème suivant : 
ThÉORÈme 4.1. Le développement par l'AJP de $\left(\alpha_{1}, \alpha_{2}\right)$ est purement périodique de longueur $l=9 m+3$ et

$$
\varepsilon=\frac{\alpha^{3}}{r}\left(\frac{\alpha}{\alpha-r c^{m}}\right)^{3 m}
$$

est une unité de $\mathbb{Z}[\alpha]$.

Preuve. L'algorithme commence avec

$$
\begin{cases}\alpha_{2}^{(0)}=\alpha, & a_{2}^{(0)}=r c^{m} \\ \alpha_{1}^{(0)}=\alpha\left(\alpha-r c^{m}\right), & a_{1}^{(0)}=c-1 .\end{cases}
$$

On établit sans difficulté les formules suivantes, pour $0 \leq s \leq m-1$ :

$$
\begin{aligned}
& \begin{cases}\alpha_{2}^{(3 s+1)}=\frac{\alpha}{r c^{m-s}}, & a_{2}^{(3 s+1)}=c^{s}, \\
\alpha_{1}^{(3 s+1)}=\frac{\alpha\left(\alpha-r c^{m}\right)}{r c^{m-s}}, & a_{1}^{(3 s+1)}=0,\end{cases} \\
& \begin{cases}\alpha_{2}^{(3 s+2)}=\frac{r c^{m-s}}{\alpha\left(\alpha-r c^{m}\right)}, & a_{2}^{(3 s+2)}=r c^{m-s-1}, \\
\alpha_{1}^{(3 s+2)}=\frac{1}{\alpha}, & a_{1}^{(3 s+2)}=0,\end{cases} \\
& \begin{cases}\alpha_{2}^{(3 s+3)}=\alpha, & a_{2}^{(3 s+3)}=r c^{m}, \\
\alpha_{1}^{(3 s+3)}=\frac{r c^{m-s-1}}{\alpha}, & a_{1}^{(3 s+3)}=0 .\end{cases}
\end{aligned}
$$

Ainsi au rang $3 m+1$ on obtient

$$
\begin{cases}\alpha_{2}^{(3 m+1)}=\frac{\alpha}{r}, & a_{2}^{(3 m+1)}=c^{m}, \\ \alpha_{1}^{(3 m+1)}=\frac{\alpha\left(\alpha-r c^{m}\right)}{r}, & a_{1}^{(3 m+1)}=\frac{c-1}{r} .\end{cases}
$$

On montre facilement les formules suivantes, pour $0 \leq s \leq m-1$ :

$$
\begin{aligned}
& \begin{cases}\alpha_{2}^{(3 s+3 m+2)}=\frac{\alpha}{c^{m-s}}, & a_{2}^{(3 s+3 m+2)}=r c^{s}, \\
\alpha_{1}^{(3 s+3 m+2)}=\frac{\alpha\left(\alpha-r c^{m}\right)}{r c^{m-s}}, & a_{1}^{(3 s+3 m+2)}=0,\end{cases} \\
& \begin{cases}\alpha_{2}^{(3 s+3 m+3)}=\frac{r c^{m-s}}{\alpha\left(\alpha-r c^{m}\right)}, & a_{2}^{(3 s+3 m+3)}=r c^{m-s-1}, \\
\alpha_{1}^{(3 s+3 m+3)}=\frac{r}{\alpha}, & a_{1}^{(3 s+3 m+3)}=0,\end{cases} \\
& \left\{\begin{array}{rlrl}
\alpha_{2}^{(3 s+3 m+4)} & =\frac{\alpha}{r}, & & a_{2}^{(3 s+3 m+4)}=c^{m}, \\
\alpha_{1}^{(3 s+3 m+4)}=\frac{c^{m-s-1}}{\alpha}, & a_{1}^{(3 s+3 m+4)}=0,
\end{array}\right.
\end{aligned}
$$


Ainsi au rang $6 m+2$ on obtient

On a ensuite les formules pour $0 \leq s \leq m-1$ :

$$
\begin{cases}\alpha_{2}^{(6 m+2)}=\alpha, & a_{2}^{(6 m+2)}=r c^{m}, \\ \alpha_{1}^{(6 m+2)}=\frac{\alpha\left(\alpha-r c^{m}\right)}{r}, & a_{1}^{(6 m+2)}=\frac{c-1}{r} .\end{cases}
$$

$$
\begin{aligned}
& \begin{cases}\alpha_{2}^{(3 s+6 m+3)}=\frac{\alpha}{c^{m-s}}, & a_{2}^{(3 s+6 m+3)}=r c^{s}, \\
\alpha_{1}^{(3 s+6 m+3)}=\frac{\alpha\left(\alpha-r c^{m}\right)}{c^{m-s}}, & a_{1}^{(3 s+6 m+3)}=0,\end{cases} \\
& \begin{cases}\alpha_{2}^{(3 s+6 m+4)}=\frac{c^{m-s}}{\alpha\left(\alpha-r c^{m}\right)}, & a_{2}^{(3 s+6 m+4)}=c^{m-s-1}, \\
\alpha_{1}^{(3 s+6 m+4)}=\frac{1}{\alpha}, & a_{1}^{(3 s+6 m+4)}=0,\end{cases} \\
& \begin{cases}\alpha_{2}^{(3 s+6 m+5)}=\alpha, & a_{2}^{(3 s+6 m+5)}=r c^{m}, \\
\alpha_{1}^{(3 s+6 m+5)}=\frac{c^{m-s-1}}{\alpha}, & a_{1}^{(3 s+6 m+5)}=0 .\end{cases}
\end{aligned}
$$

Finalement,

$$
\left\{\begin{array}{l}
\alpha_{2}^{(9 m+3)}=\alpha=\alpha_{2}^{(0)}, \\
\alpha_{1}^{(9 m+3)}=\alpha\left(\alpha-r c^{m}\right)=\alpha_{1}^{(0)} .
\end{array}\right.
$$

Ainsi, ce développement est purement périodique de longueur $l=3(3 m+1)$ et une unité de $\mathbb{Z}[\alpha]$ est donnée par la formule de Hasse-Bernstein [2], à savoir :

$$
\varepsilon=\prod_{r=0}^{l-1} \alpha_{2}^{(r)}=\frac{\alpha^{3}}{r}\left(\frac{\alpha}{\alpha-r c^{m}}\right)^{3 m} .
$$

Ceci termine la démonstration du théorème 4.1.

4.2. Développement par l'algorithme de Voronoï. Avec les mêmes notations que celles définies précédemment, à savoir $L=\left\langle 1, \alpha_{1}, \alpha_{2}\right\rangle$ est un réseau de $K$ et $\psi=u+v \alpha_{1}+w \alpha_{2}$ est le point extrémal adjacent à 1 dans $L$, on a les lemmes suivants :

Lemme 4.2. Pour un entier $s, 0 \leq s \leq m$,

- si $L=\left\langle 1, \alpha-r c^{m}, c^{s} / \alpha\right\rangle$ alors $(u, v, w)=\left(r c^{m}, 1,0\right)$;

- si $L=\left\langle 1,\left(\alpha-r c^{m}\right) / r, c^{s} / \alpha\right\rangle$ alors $(u, v, w)=\left(c^{m}, 1,0\right)$;

- si $L=\left\langle 1, \alpha-r c^{m}, r c^{s} / \alpha\right\rangle$ alors $(u, v, w)=\left(r c^{m}, 1,0\right)$.

Lemme 4.3. Pour un entier $s, 0 \leq s \leq m-1$,

- si $L=\left\langle 1, c^{s} / \alpha^{2}, 1 / \alpha\right\rangle$ alors $(u, v, w)=\left(c^{s}, 1,0\right)$;

- si $L=\left\langle 1, r c^{s} / \alpha^{2}, r / \alpha\right\rangle$ alors $(u, v, w)=\left(r c^{s}, 1,0\right)$;

- si $L=\left\langle 1, r c^{s} / \alpha^{2}, 1 / \alpha\right\rangle$ alors $(u, v, w)=\left(r c^{s}, 1,0\right)$. 
Lemme 4.4. Pour un entier $s, 0 \leq s \leq m-1$,

- si $L=\left\langle 1, \frac{\alpha-r c^{m}}{c^{s+1}}, \frac{\alpha\left(\alpha-r c^{m}\right)}{c^{s+1}}\right\rangle$ alors $(u, v, w)=\left(r c^{m-1-s}, 1,0\right)$;

- si $L=\left\langle 1, \frac{\alpha-r c^{m}}{c^{s+1}}, \frac{\alpha\left(\alpha-r c^{m}\right)}{r c^{s+1}}\right\rangle$ alors $(u, v, w)=\left(r c^{m-1-s}, 1,0\right)$;

- si $L=\left\langle 1, \frac{\alpha-r c^{m}}{r c^{s+1}}, \frac{\alpha\left(\alpha-r c^{m}\right)}{r c^{s+1}}\right\rangle$ alors $(u, v, w)=\left(c^{m-1-s}, 1,0\right)$.

Ces lemmes sont encore semblables à ceux énoncés dans [1], qui correspondent à $r=1$, et les démonstrations en sont identiques.

A l'aide de ces lemmes on procède de même que pour la première famille et on détermine la suite des points extrémaux de $\mathcal{O}$. On obtient ainsi, pour $0 \leq s \leq m-1$, les résultats donnés dans le tableau 2 .

\section{Tableau 2}

\begin{tabular}{cccc}
\hline$k$ & $L_{k}=\left\langle 1, \phi_{k} / \psi_{k}, \psi_{k-1} / \psi_{k}\right\rangle$ & $\psi_{k+1} / \psi_{k}$ & $\phi_{k+1} / \psi_{k}$ \\
\hline 0 & $\left\langle 1, \alpha-r c^{m}, r c^{m} / \alpha\right\rangle$ & $\left(r c^{m}, 1,0\right)$ & $(c-1,0,1)$ \\
$\vdots$ & $\vdots$ & $\vdots$ & $\vdots$ \\
$3 s+1$ & $\left\langle 1, \alpha-r c^{m}, c^{s} / \alpha\right\rangle$ & $\left(r c^{m}, 1,0\right)$ & $(0,0,1)$ \\
$3 s+2$ & $\left\langle 1, c^{s} / \alpha^{2}, 1 / \alpha\right\rangle$ & $\left(c^{s}, 1,0\right)$ & $(0,0,1)$ \\
$3 s+3$ & $\left\langle 1,\left(\alpha-r c^{m}\right) / c^{s+1}, \alpha\left(\alpha-r c^{m}\right) / c^{s+1}\right\rangle$ & $\left(r c^{m-1-s}, 1,0\right)$ & $(0,0,1)$ \\
$\vdots$ & $\vdots$ & $\vdots$ & $\vdots$ \\
$3 m+1$ & $\left\langle 1, \alpha-r c^{m}, c^{m} / \alpha\right\rangle$ & $\left(r c^{m}, 1,0\right)$ & $\left(\frac{c-1}{r}, 0,1\right)$ \\
$\vdots$ & $\vdots$ & $\vdots$ & $\vdots$ \\
$3 s+3 m+2$ & $\left\langle 1,\left(\alpha-r c^{m}\right) / r, c^{s} / \alpha\right\rangle$ & $\left(c^{m}, 1,0\right)$ & $(0,0,1)$ \\
$3 s+3 m+3$ & $\left\langle 1, r c^{s} / \alpha^{2}, r / \alpha\right\rangle$ & $\left(r c^{s}, 1,0\right)$ & $(0,0,1)$ \\
$3 s+3 m+4\left\langle 1,\left(\alpha-r c^{m}\right) / c^{s+1}, \alpha\left(\alpha-r c^{m}\right) / c^{s+1}\right\rangle$ & $\left(r c^{m-1-s}, 1,0\right)$ & $(0,0,1)$ \\
$\vdots$ & $\vdots$ & $\vdots$ & $\vdots$ \\
$6 m+2$ & $\left\langle 1,\left(\alpha-r c^{m}\right) / r, c^{m} / \alpha\right\rangle$ & $\left(c^{m}, 1,0\right)$ & $\left(\frac{c-1}{r}, 0,1\right)$ \\
$\vdots$ & $\vdots$ & $\vdots$ & $\vdots$ \\
$6 m+3 s+3$ & $\left\langle 1, \alpha-r c^{m}, r c^{s} / \alpha\right\rangle$ & $\left(r c^{m}, 1,0\right)$ & $(0,0,1)$ \\
$6 m+3 s+4$ & $\left\langle 1, r c^{s} / \alpha^{2}, 1 / \alpha\right\rangle$ & $\left(r c^{s}, 1,0\right)$ & $(0,0,1)$ \\
$6 m+3 s+5$ & $\left\langle 1, \frac{\alpha-r c^{m}}{r c^{s+1}}, \frac{\alpha\left(\alpha-r c^{m}\right)}{r c^{s+1}}\right\rangle$ & $\left(r c^{m-1-s}, 1,0\right)$ & $(0,0,1)$ \\
\hline & & &
\end{tabular}


On a noté

$$
\phi_{0}=\alpha-r c^{m}, \quad \psi_{-1}=r c^{m} / \alpha .
$$

De même que précédemment, on en déduit que

$$
\psi_{9 m+3}=\frac{\alpha^{3}}{r}\left(\frac{\alpha}{\alpha-r c^{m}}\right)^{3 m} .
$$

On a

$$
N\left(\psi_{9 m+3}\right)=1 \text { et } N\left(\psi_{i}\right) \neq 1 \text { si } 0<i \leq 9 m+2 .
$$

Donc $\psi_{9 m+3}$ est l'unité fondamentale supérieure à 1 de $\mathcal{O}$ et la longueur de la période du développement de l'algorithme de Voronoï est $l=9 m+3$.

On peut encore remarquer que :

- les points auxiliaires choisis sont donnés par l'AJP;

- les développements par l'AJP et par l'algorithme de Voronoï cö̈ncident : ils ont donc la même longueur et l'unité obtenue par l'AJP est l'unité fondamentale supérieure à 1 de $\mathcal{O}$.

5. Généralisation de ces familles. Soit $c \geq 2, m \geq 1,1 \leq t \leq m$ et $r$ des entiers tel que $r \neq 1$ et $r$ divise $c^{d}-1$, où $d=\operatorname{pgcd}(m, t)$. On considère le polynôme

$$
f(X)=X^{3}-r c^{m} X^{2}-\left(c^{t}-1\right) X-r c^{m} .
$$

On montre toujours facilement que $f(X)$ est irréductible et admet une racine réelle unique notée $\alpha$ telle que $r c^{m}<\alpha<r c^{m}+1$.

On note $K=\mathbb{Q}(\alpha), \mathcal{O}=\mathbb{Z}[\alpha], \alpha_{2}=\alpha, \alpha_{1}=\alpha\left(\alpha-r c^{m}\right)$.

Cette famille de corps, dépendant de quatre paramètres, généralise les deux familles précédentes. On obtient de manière analogue les deux théorèmes suivants :

THÉORÈME 5.1. Le développement par l'AJP de $\left(\alpha_{1}, \alpha_{2}\right)$ est purement périodique de longueur $l=3(3 m+t) / d$ et

$$
\varepsilon=\frac{\alpha^{3 t / d}}{r}\left(\frac{\alpha}{\alpha-r c^{m}}\right)^{3 m / d}
$$

est une unité de $\mathbb{Z}[\alpha]$.

Pour démontrer ce théorème il suffit de combiner les démonstrations des deux théorèmes correspondants pour les familles étudiées précédemment.

ThÉORÈme 5.2. L'unité fondamentale supérieure à 1 de $\mathcal{O}$ est

$$
\varepsilon=\frac{\alpha^{3 t / d}}{r}\left(\frac{\alpha}{\alpha-r c^{m}}\right)^{3 m / d}
$$

et la longueur du développement par l'algorithme de Voronoï est $l=$ $3(3 m+t) / d$. 
Pour les mêmes raisons que plus haut, nous ne détaillons pas la démonstration de ce théorème.

\section{Références}

[1] B. Adam, Voronoï-algorithm expansion of two families with period length going to infinity, Math. Comp. 64 (1995), 1687-1704.

[2] L. Bernstein, The Jacobi-Perron Algorithm, its Theory and Application, Lecture Notes in Math. 207, Springer, Berlin, 1971.

[3] B. N. Delone and D. K. Faddeev, The Theory of Irrationalities of the Third Degree, Transl. Math. Monographs 10, Amer. Math. Soc., Providence, R.I., 1964.

[4] E. Dubois et A. Farhane, Unité fondamentale dans des familles d'ordres cubiques, Utilitas Math. 47 (1995), 97-115.

[5] A. Farhane, Spécialisation de points extrémaux. Application aux fractions continues et aux unités d'une famille de corps cubiques, thèse, Caen, 1992.

[6] F. Halter-Koch, Einige periodische Kettenbruchentwicklungen und Grundeinheiten quadratischer Ordnungen, Abh. Math. Sem. Univ. Hamburg 59 (1989), 157-169.

[7] C. Levesque and G. Rhin, Two families of periodic Jacobi algorithms with period lengths going to infinity, J. Number Theory 37 (1991), 173-180.

[8] R. A. Mollin and H. C. Williams, Consecutive powers in continued fractions, Acta Arith. 61 (1992), 233-264.

[9] O. Perron, Grundlagen für eine Theorie des Jacobischen Kettenbruchalgorithmus, Math. Ann. 64 (1907), 1-76.

[10] D. Shanks, On Gauss's class number problems, Math. Comp. 23 (1969), 151-163.

[11] G. F. Voronoï, On a generalization of the algorithm of continued fractions, Doctoral Dissertation, Warszawa, 1896 (en russe).

[12] H. C. Williams, Some generalizations of the $S_{n}$ sequence of Shanks, Acta Arith. 69 (1995), 199-215.

Département de Mathématiques

URA CNRS 399

UFR MIM, Université de Metz, Ile du Saulcy

57045 Metz Cedex 01, France

E-mail: adam@poncelet.univ-metz.fr 